\title{
On the Relation Between Casimir Forces and Bulk Correlations
}

\author{
Marek Napiórkowski · Jarosław Piasecki
}

Received: 2 April 2014 / Accepted: 10 June 2014 / Published online: 2 July 2014

(C) The Author(s) 2014. This article is published with open access at Springerlink.com

\begin{abstract}
Within a microscopic approach we show that in the case of an ideal quantum gas enclosed in a slit the Casimir force can be simply expressed in terms of the bulk one-particle density matrix. The corresponding formula, which holds both for bosons and fermions, allows to relate the range of the Casimir force to the bulk correlation length. The low-temperature behavior of the Casimir forces is derived.
\end{abstract}

\section{Introduction}

The Casimir forces acting on planar walls immersed in a perfect quantum gas (the slit geometry) have been the object of numerous studies [1-17]. However, a microscopic explanation of the remarkable similarity in the behavior of the range of the Casimir forces and the bulk correlation length remained on open problem. Our aim here is to clarify this physically important and intriguing point.

We will show that although the Casimir forces depend on the boundary conditions imposed by the confining walls, they can be simply expressed in terms of the one-particle density matrix $[1,18]$ calculated in the thermodynamic limit

$$
<\mathbf{r}_{1}\left|\hat{\rho}_{1}\right| \mathbf{r}_{2}>\equiv \rho_{1}\left(r_{12}\right)
$$

which itself does not depend on the boundary conditions. Here $r_{12}=\left|\mathbf{r}_{1}-\mathbf{r}_{2}\right|$ denotes the distance between two space points $\mathbf{r}_{1}$ and $\mathbf{r}_{2}$. The number density $\rho$ of a homogeneous gas is given by $\rho=\rho_{1}(0)$.

The two-particle number density $\rho_{2}\left(r_{12}\right)$ of pairs of particles separated by distance $r_{12}$ is given by diagonal elements of the two-particle reduced density matrix $[1,6,18]$

$$
<\mathbf{r}_{1}, \mathbf{r}_{2}\left|\hat{\rho}_{2}\right| \mathbf{r}_{1}, \mathbf{r}_{2}>\equiv \rho_{2}\left(r_{12}\right)
$$

M. Napiórkowski $(\bowtie) \cdot$ J. Piasecki

Faculty of Physics, Institute of Theoretical Physics, University of Warsaw, Hoża 69,

00-681 Warsaw, Poland

e-mail: marnap@fuw.edu.pl 
The deviation of $\rho_{2}$ from $\rho^{2}$ defines the pair correlation function

$$
\chi_{2}(r)=\rho_{2}(r)-\rho^{2} .
$$

In the case of a perfect Bose gas the correlation function $\chi_{2}^{B}(r)$ can be expressed exclusively in terms of $\rho_{1}^{B}(r)[1]$

$$
\chi_{2}^{B}(r)=\left\{\begin{array}{ccc}
{\left[\rho_{1}^{B}(r)\right]^{2}} & \text { if } & \rho<\rho_{c} \\
{\left[\rho_{1}^{B}(r)\right]^{2}-\left(\rho-\rho_{c}\right)^{2}} & \text { if } & \rho \geq \rho_{c},
\end{array}\right.
$$

where $\rho_{c}$ denotes the critical density of the Bose-Einstein condensation. Note that the form of the function $\rho_{1}^{B}(r)$ is different for $\rho<\rho_{c}$ and for $\rho>\rho_{c}$.

Even a simpler relation holds for a perfect Fermi gas [18]

$$
\chi_{2}^{F}(r)=-\left[\rho_{1}^{F}(r)\right]^{2} .
$$

The explicit expressions for $\rho_{1}^{B}(r)$ and $\rho_{1}^{F}(r)$ will be discussed later on.

The derivation of an exact analytic relation between the one-particle function $\rho_{1}(r)$ describing an infinite system and the Casimir forces is presented in Sect. 2. The derived formula forms the basis of the subsequent analysis. Most importantly, it permits to establish an explicit relation between the pair correlation function $\chi(r)$ and the Casimir forces. Indeed, the fact that the Casimir forces can be expressed in terms of the one-particle density matrix implies their direct relation to the correlation function through Eqs. (4) and (5) (when writing these equations we do not display their spin dependence). Consequently, the analogy in the behavior of the range of the forces and the bulk correlation length can be clearly explained within a microscopic approach (Sect. 3). Our analysis permits to recover in a concise way various specific results derived elsewhere by different techniques.

\section{Relation Between Casimir Forces and the One-Particle Density Matrix}

We consider a perfect gas composed of identical particles of mass $m$ contained in $L \times L \times D$ rectangular box of volume $V=L^{2} D$. We choose the coordinate frame whose axes indexed by $x, y, z$ are perpendicular to the walls of the box. In particular, the $z$ axis is oriented perpendicularly to the square walls.

In the case of bosons the grand canonical free energy $\Omega^{B}(T, L, D, \mu)$ at temperature $T$ and chemical potential $\mu$ is given by the series

$$
\Omega^{B}(T, L, D, \mu)=k_{B} T \sum_{\mathbf{k}} \ln \left\{1-\mathbb{Z} \exp \left(-\frac{\lambda^{2}}{4 \pi} \mathbf{k}^{2}\right)\right\}
$$

whereas for fermions

$$
\Omega^{F}(T, L, D, \mu)=-k_{B} T \sum_{\mathbf{k}} \ln \left\{1+\mathbb{Z} \exp \left(-\frac{\lambda^{2}}{4 \pi} \mathbf{k}^{2}\right)\right\} .
$$

Here $\lambda=h / \sqrt{2 \pi m k_{B} T}$ denotes the de Broglie thermal wavelength, $\mathbb{z}=\exp \left(\mu / k_{B} T\right)$, and $k_{B}$ is the Boltzmann constant. The summation in the above equations spreads over the wave vectors $\mathbf{k}$ allowed by the boundary conditions. As already mentioned in the Introduction, to simplify the notation we do not take into account the spin of the particles.

We adopt periodic boundary conditions in $x$ and $y$ directions so that the wave vector components $k_{x}$ and $k_{y}$ take the values $2 \pi n / L$, where $n=0, \pm 1, \pm 2, \ldots$. Dividing then 
Eq. (6) by $L^{2}$, and taking the limit $L \rightarrow \infty$ of infinitely extended square walls we get the formula for the total grand canonical free-energy density per unit wall area

$$
\begin{aligned}
& \omega^{B}(T, D, \mu)=\lim _{L \rightarrow \infty} \frac{\Omega^{B}(T, L, D, \mu)}{L^{2}} \\
& =k_{B} T \sum_{k_{z}} \iint \frac{d k_{x} d k_{y}}{(2 \pi)^{2}} \ln \left\{1-\mathbb{Z} \exp \left[-\frac{\lambda^{2}}{4 \pi}\left(k_{x}^{2}+k_{y}^{2}+k_{z}^{2}\right)\right]\right\} .
\end{aligned}
$$

The corresponding formula for fermions reads

$$
\omega^{F}(T, D, \mu)==-k_{B} T \sum_{k_{z}} \iint \frac{d k_{x} d k_{y}}{(2 \pi)^{2}} \ln \left\{1+\mathbb{Z} \exp \left[-\frac{\lambda^{2}}{4 \pi}\left(k_{x}^{2}+k_{y}^{2}+k_{z}^{2}\right)\right]\right\} .
$$

The Dirichlet boundary conditions are appropriate for hard walls. However, for completness, we will consider three types of boundary conditions in the $z$-direction:

1. periodic, $k_{z}=2 \pi n / D n=0, \pm 1, \pm 2, \ldots$

2. Dirichlet, $k_{z}=\pi n / D, n=1,2, \ldots$

3. Neumann, $k_{z}=\pi n / D, n=0,1,2, \ldots$.

There exist simple relations between the energy densities corresponding to the above boundary conditions. It can be readily checked that

$$
\omega_{\text {per }}^{B}(T, 2 D, \mu)=\sigma^{B}(T, \mu)+2 \omega_{D i r}^{B}(T, D, \mu)=-\sigma^{B}(T, \mu)+2 \omega_{N e u}^{B}(T, D, \mu)
$$

where

$$
\sigma^{B}(T, \mu)=k_{B} T \iint \frac{d k_{x} d k_{y}}{(2 \pi)^{2}} \ln \left\{1-\mathbb{Z} \exp \left[-\frac{\lambda^{2}}{4 \pi}\left(k_{x}^{2}+k_{y}^{2}\right)\right]\right\}=-\frac{k_{B} T}{\lambda^{2}} g_{2}\left(e^{\mu / k_{B} T}\right)
$$

corresponds to the contribution from $k_{z}=0$ in the series appearing in Eq. (8). The Bose function $g_{2}$ is defined in the standard way: $g_{2}(\mathbb{Z})=\sum_{l=1}^{\infty} \mathbb{Z}^{l} / l^{2}$. We note that $\sigma^{B}(T, \mu) / 4=$ $\gamma_{N e u}^{B}=-\gamma_{D i r}^{B}(T, \mu)$, where $\gamma_{N e u, D i r}^{B}(T, \mu)$ denotes the coefficient of surface tension of an ideal Bose gas corresponding to the Neumann or Dirichlet boundary conditions, respectively [13].

Similarly, for fermions we find

$$
\omega_{\text {per }}^{F}(T, 2 D, \mu)=\sigma^{F}(T, \mu)+2 \omega_{D i r}^{F}(T, D, \mu)=-\sigma^{F}(T, \mu)+2 \omega_{N e u}^{F}(T, D, \mu),
$$

where

$\sigma^{F}(T, \mu)=-k_{B} T \iint \frac{d k_{x} d k_{y}}{(2 \pi)^{2}} \ln \left\{1+\mathbb{Z} \exp \left[-\frac{\lambda^{2}}{4 \pi}\left(k_{x}^{2}+k_{y}^{2}\right)\right]\right\}=\frac{k_{B} T}{\lambda^{2}} g_{2}\left(-e^{\mu / k_{B} T}\right)$.

As in the bosonic case we identify the expressions for the coefficients of the surface tension of an ideal Fermi gas : $\gamma_{D i r}^{F}(T, \mu)=-\gamma_{N e u}^{F}(T, \mu)=-k_{B} T g_{2}\left(-e^{\mu / k_{B} T}\right) / 4 \lambda^{2}$.

We now turn to the derivation of the formula for the Casimir force $F(T, D, \mu)$ (or Casimir pressure) relating it to the one-particle density matrix. By definition

$$
F(T, D, \mu)=-\frac{\partial}{\partial D} \omega_{s}(T, D, \mu),
$$


where $\omega_{s}(T, D, \mu)$ is the excess free energy density

$$
\omega_{s}(T, D, \mu)=\omega(T, D, \mu)-D \omega_{b}(T, \mu)
$$

equal to the difference between the total grand canonical free energy density per unit wall area $\omega(T, D, \mu)$ and the bulk free energy density $\omega_{b}(T, \mu)$ (evaluated in the thermodynamic limit) multiplied by $D$.

We begin by evaluating the density $\omega^{B}(T, D, \mu)$, see Eq. (8), under the periodic boundary conditions

$$
\begin{aligned}
& \omega_{\text {per }}^{B}(T, D, \mu)=k_{B} T \sum_{n=-\infty}^{+\infty} \iint \frac{d k_{x} d k_{y}}{(2 \pi)^{2}} \ln \left\{1-\mathbb{Z} \exp \left(-\frac{\lambda^{2}}{4 \pi}\left[k_{x}^{2}+k_{y}^{2}+\left(\frac{2 \pi n}{D}\right)^{2}\right]\right)\right\} \\
& =\sigma^{B}(T, \mu)+2 k_{B} T \sum_{n=1}^{+\infty} \iint \frac{d k_{x} d k_{y}}{(2 \pi)^{2}} \ln \left\{1-\mathbb{Z} \exp \left(-\frac{\lambda^{2}}{4 \pi}\left[k_{x}^{2}+k_{y}^{2}+\left(\frac{2 \pi n}{D}\right)^{2}\right]\right)\right\} .
\end{aligned}
$$

At this point we will use the non-expanded form of the Euler-Maclaurin formula [14]

$$
\sum_{n=1}^{N} f(n)=\int_{0}^{N} f(x) d x+\frac{1}{2}[f(N)-f(0)]+\int_{0}^{N}\left(x-[x]-\frac{1}{2}\right) f^{\prime}(x) d x,
$$

where $[x]$ denotes the largest integer not exceeding $x$, and $f^{\prime}(x)$ is the derivative of $f(x)$. The function $(x-[x]-1 / 2)$ appearing in the integrand above is periodic and has the following Fourier series representation

$$
x-[x]-\frac{1}{2}=-\frac{1}{\pi} \sum_{p=1}^{\infty} \frac{\sin (2 p \pi x)}{p} .
$$

Using Eqs. (17) and (18) we find

$$
\begin{aligned}
& \omega_{\text {per }}^{B}(T, D, \mu)-D \omega_{b}(T, \mu) \\
& =2 k_{B} T \iint \frac{d k_{x} d k_{y}}{(2 \pi)^{2}} \int_{0}^{\infty} d x\left(x-[x]-\frac{1}{2}\right) \frac{\partial}{\partial x} \ln \left\{1-\mathbb{Z} \exp \left(-\frac{\lambda^{2}}{4 \pi}\left[k_{x}^{2}+k_{y}^{2}+\left(\frac{2 \pi x}{D}\right)^{2}\right]\right)\right\} \\
& =\lambda^{2} k_{B} T \iiint \frac{d \mathbf{k}}{(2 \pi)^{3}}\left(\frac{D k_{z}}{2 \pi}-\left[\frac{D k_{z}}{2 \pi}\right]-\frac{1}{2}\right) \frac{k_{z}}{\mathbb{Z}^{-1} \exp \left(\lambda^{2} \mathbf{k}^{2} / 4 \pi\right)-1} \\
& =-\frac{h^{2}}{2 \pi^{2} m} \sum_{p=1}^{\infty} \iiint \frac{d \mathbf{k}}{(2 \pi)^{3}} \frac{\sin \left(p D k_{z}\right)}{p} k_{z} \rho^{B}(\mathbf{k})=\frac{h^{2}}{2 \pi^{2} m} \sum_{p=1}^{\infty} \frac{\partial}{\partial D} \iiint \frac{d \mathbf{k}}{(2 \pi)^{3}} \frac{\cos \left(p D k_{z}\right)}{p^{2}} \rho^{B}(\mathbf{k})
\end{aligned}
$$

where $d \mathbf{k}=d k_{x} d k_{y} d k_{z}$, and $\rho^{B}(\mathbf{k}) \equiv \rho^{B}(k), k=|\mathbf{k}|$, is the mean occupation number of the one-particle state $\mathbf{k}$.

The inverse Fourier transform of $\rho^{B}(\mathbf{k})$ is equal to the one-particle density matrix

$$
\rho_{1}^{B}(r)=\iiint \frac{d \mathbf{k}}{(2 \pi)^{3}} e^{i \mathbf{k} \cdot \mathbf{r}} \rho^{B}(k)=\iiint \frac{d \mathbf{k}}{(2 \pi)^{3}} \cos (\mathbf{k} \cdot \mathbf{r}) \rho^{B}(k) .
$$

We thus get from (19) the relation

$$
\omega_{p e r}^{B}(T, D, \mu)-D \omega_{b}(T, \mu)=\frac{h^{2}}{2 \pi^{2} m} \frac{\partial}{\partial D} \sum_{p=1}^{\infty} \frac{\rho_{1}^{B}(p D)}{p^{2}} .
$$


Finally, using the defining Eq. (14) we arrive at the basic formula

$$
F_{p e r}^{B}(T, D, \mu)=-\frac{h^{2}}{2 \pi^{2} m} \frac{\partial^{2}}{\partial D^{2}} \sum_{p=1}^{\infty} \frac{\rho_{1}^{B}(p D)}{p^{2}}
$$

which relates the Casimir force to the one-particle density matrix. Although the above basic formula has been derived for the ideal Bose gas we expect a similar formula to hold for the imperfect Bose gas $[13,15,17,19,20]$, where the interparticle repulsion is taken into account in the mean-field way. On the other hand, one should not expect such simple relation to hold between the Casimir force and the one-particle density matrix when fluctuations of the interacting Bose gas are taken into account.

Similar calculation performed for a perfect Fermi gas leads to an identical relation between the Casimir force and the one-particle density matrix $\rho_{1}^{F}(p D)$

$$
F_{p e r}^{F}(T, D, \mu)=-\frac{h^{2}}{2 \pi^{2} m} \frac{\partial^{2}}{\partial D^{2}} \sum_{p=1}^{\infty} \frac{\rho_{1}^{F}(p D)}{p^{2}} .
$$

Before ending this section we notice that Eqs. (10) and (12) immediately lead to relations between the Casimir forces corresponding to different boundary conditions:

$$
F_{D i r}(T, D, \mu)=F_{N e u}(T, D, \mu)=F_{p e r}(T, 2 D, \mu) .
$$

The above equalities hold both for bosons and fermions. We note here that in the case of non-planar walls the dependence of the Casimir forces on the boundary conditions becomes more involved, see [5,21-23].

\section{Range of Casimir Forces and Bulk Correlation Length}

The large-distance behavior of the one-particle density matrix of a Bose gas in the absence of condensate $\left(\mu<0\right.$, or $\left.\rho<\rho_{c}\right)$ is governed by an exponential law [1]

$$
\rho_{1}^{B}(r) \sim \frac{1}{r \lambda^{2}} \exp \left[-\frac{2 r}{\lambda} \sqrt{\frac{\pi(-\mu)}{k_{B} T}}\right] .
$$

The basic Eq. (22) implies thus an exponential decay of the attractive Casimir force, see Fig. 1, with the range-in the case of periodic boundary conditions-given by

$$
\kappa_{p e r}^{B}=\frac{\lambda}{2} \sqrt{\frac{k_{B} T}{\pi(-\mu)}}=\frac{\hbar}{\sqrt{2 m(-\mu)}} .
$$

Using Eq. (24) we readily get the ranges corresponding to the Dirichlet and Neumann boundary conditions

$$
\kappa_{D i r}^{B}=\kappa_{N e u}^{B}=\frac{1}{2} \kappa_{p e r}^{B}=\frac{\hbar}{2 \sqrt{2 m(-\mu)}} .
$$

Equations (26) and (27) show that upon approaching the phase containing the condensate $(\mu \rightarrow 0)$ the range of the force diverges with the critical exponent $v=1 / 2$.

In the two-phase region $(\mu=0)$ there appears an off-diagonal long range order, and the one-particle density matrix $\rho_{1}^{B}(r)$ approaches for $r \rightarrow \infty$ a nonzero value $\left(\rho-\rho_{c}\right)$ following 


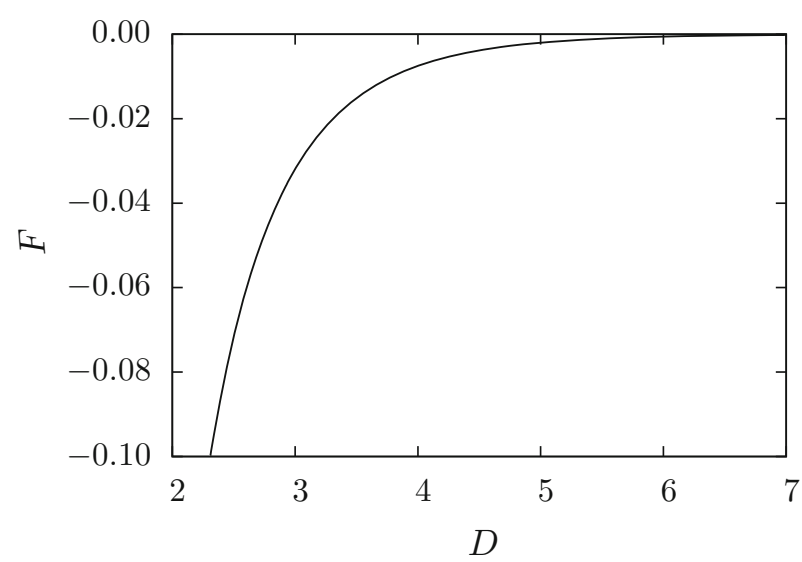

Fig. 1 The Casimir force for the ideal Bose gas as function of the distance bewteen the walls. The Casimir force is evaluated in $2 \hbar^{2} \lambda^{-2} \kappa^{-3} m^{-1}$ units. The distance is expressed in $\kappa$ units

the power law [1]

$$
\rho_{1}^{B}(r)-\left(\rho-\rho_{c}\right) \approx \frac{1}{r \lambda^{2}} .
$$

According to Eq. (22) the corresponding decay of the Casimir force for $D \gg \lambda$ is given by

$$
F_{p e r}^{B}(T, D, 0)=-\frac{h^{2}}{2 \pi^{2} m} \frac{\partial^{2}}{\partial D^{2}}\left[\sum_{p=1}^{\infty} \frac{1}{p^{3} D \lambda^{2}}\right]=-\frac{2 k_{B} T \zeta(3)}{\pi D^{3}},
$$

where $\zeta(3)=\sum_{p=1}^{\infty} p^{-3}$ is the Riemann zeta function. We note that in the two-phase regime the ratio of the Casimir force $F_{p e r}^{B}(T, D, 0)$ to the bulk pressure of the ideal Bose gas $p=k_{B} T \zeta(5 / 2) / \lambda^{3}$ is for large distances $D \gg \lambda$ proportional to $(\lambda / D)^{3}$ and thus small. Using Eq. (24) we also readily find $[8,9]$

$$
F_{D i r}^{B}(T, D, 0)=F_{N e u}^{B}(T, D, \mu)=-\frac{k_{B} T \zeta(3)}{4 \pi D^{3}} .
$$

The behavior of the correlation function follows directly from Eq. (4). In the one-phase region $(\mu<0)$ the correlation function $\chi_{2}^{B}(r)$ is equal to $\left[\rho_{1}^{B}(r)\right]^{2}$. Correlations are thus exponentially damped with the bulk correlation length $\xi_{B}$ such that

$$
\xi^{B}=\kappa_{D i r}^{B}=\kappa_{N e u}^{B}=\frac{1}{2} \kappa_{p e r}^{B}
$$

The correlation length $\xi^{B}$ is exactly equal to the range of Casimir forces under Dirichlet and Neumann boundary conditions. ${ }^{1}$ Our basic Eq. (22) sheds light on the origin of this remarkable agreement. When $\mu \rightarrow 0$, both $\xi^{B}$ and the range of Casimir forces diverge with the same critical exponent 1/2. In Ref.[15] analogous results have been derived for the imperfect Bose gas $[19,20]$ which belongs to a different universality class than the ideal Bose gas [17].

In the case of a Fermi gas, although there is no phase transition, both correlations and Casimir forces are characterized by decay lengths which diverge for $T \rightarrow 0$. Equation (23)

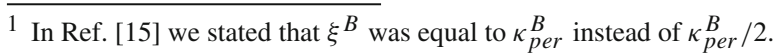


permits to establish a clear connection between the correlation length and the range of the forces.

The one-particle density matrix of a perfect Fermi gas is given by the formula [18]

$$
\rho_{1}^{F}(r)=-\frac{1}{\lambda^{3}} \sum_{j=1}^{\infty} \frac{(-\mathbb{Z})^{j}}{j^{3 / 2}} \exp \left[-\frac{\pi r^{2}}{j \lambda^{2}}\right] .
$$

We are interested in the properties of $\rho_{1}^{F}(r)$ at low temperatures where the following asymptotic formula holds [18]

$$
\left.\rho_{1}^{F}(r)\right|_{T \rightarrow 0}=-\frac{1}{\lambda^{2} k_{F} r} \frac{\partial}{\partial r}\left\{\frac{\sin \left(k_{F} r\right)}{\sinh \left(2 \pi^{2} r / \lambda^{2} k_{F}\right)}\right\}
$$

with the Fermi wavenumber $k_{F}=\left(6 \pi^{2} \rho\right)^{1 / 3}$. The large $r$ behavior involves thus exponentially damped oscillations with the characteristic decay length $\lambda^{2} k_{F}$.

Equations (23) and (24) allow to find the relation between decay lenghts corresponding to different boundary conditions with the result

$$
\kappa_{\text {Dir }}^{F}=\kappa_{N e u}^{F}=\frac{1}{2} \kappa_{p e r}^{F}=\frac{\lambda^{2} k_{F}}{4 \pi^{2}} .
$$

Taking into account that for $T>0$ the correlation function $\chi_{2}^{F}(r)=-\left[\rho_{1}^{F}(r)\right]^{2}$, we conclude that also correlations decay via exponentially damped oscillations with the correlation length

$$
\xi^{F}=\frac{\lambda^{2} k_{F}}{4 \pi^{2}}=\kappa_{D i r}^{F}=\kappa_{N e u}^{F} .
$$

We find here a complete analogy with the Bose gas, where $\xi^{B}=\kappa_{\mathrm{Dir}}^{B}=\kappa_{\mathrm{Neu}}^{B}$.

At zero temperature Eq. (33) takes the following form [18]

$$
\left.\rho_{1}^{F}(r)\right|_{T=0}=\frac{\sin \left(k_{F} r\right)-k_{F} r \cos \left(k_{F} r\right)}{2 \pi^{2} r^{3}} .
$$

Upon inserting this formula into (23) we find that for $D \rightarrow \infty$ the dominant contribution to the Casimir force takes the following form

$$
\begin{gathered}
F_{p e r}^{F}\left(0, D, \mu_{F}\right)=-\frac{\hbar^{2} k_{F}^{3}}{m \pi^{2}} \sum_{p=1}^{\infty} \frac{\cos \left(p k_{F} D\right)}{(p D)^{2}} \\
=-\frac{1}{D^{2}}\left(\frac{\hbar^{2} k_{F}^{3}}{m}\right)\left[\frac{1}{6}-\left(\frac{k_{F} D}{2 \pi}-\left[\frac{k_{F} D}{2 \pi}\right]\right)+\left(\frac{k_{F} D}{2 \pi}-\left[\frac{k_{F} D}{2 \pi}\right]\right)^{2}\right] .
\end{gathered}
$$

The Casimir force displays a typical for fermions oscillatory behavior which is superimposed on the power-law decay, see Fig. 2. Note that the damped oscillations imply the change of sign of the Casimir force: upon increasing the distance between the walls the force becomes in turn attractive and repulsive, with the infinite set of stability points. The amplitude of these oscillations increases with decreasing temperature. In the $T=0$ limit the oscillations are generated by the periodic function

$$
D \rightarrow\left(\frac{k_{F} D}{2 \pi}-\left[\frac{k_{F} D}{2 \pi}\right]\right) .
$$




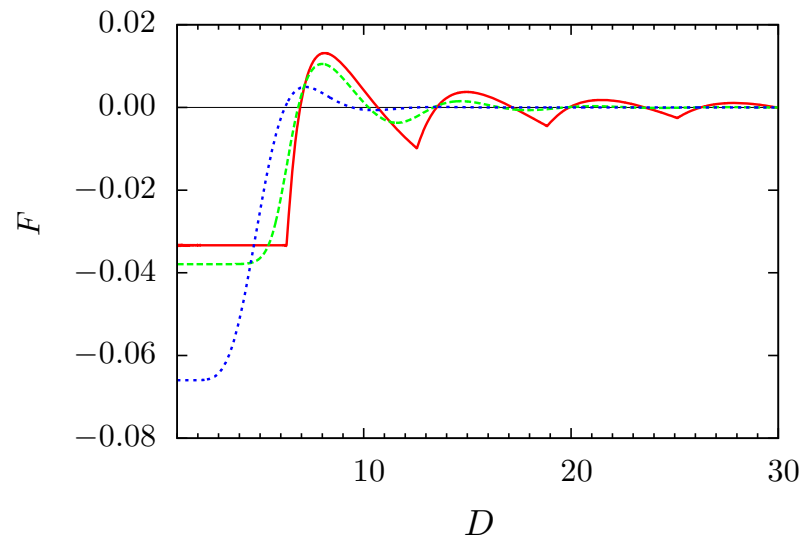

Fig. 2 The Casimir force for the ideal Fermi gas as function of the distance bewteen the walls for three different temperatures. The Casimir force is evaluated in $2 \hbar^{2} k_{F}^{5} \pi^{-2} m^{-1}$ units. The distance is expressed in $k_{F}^{-1}$ units. The dimensionless quantity $t=2 * \pi^{2} \lambda^{-2} k_{F}^{-2} m^{-1} \sim T$ is used as the measure of temperature. The dotted (blue) line corresponds to $t=0.5$, the dashed (green) line-to $t=0.2$, and the solid (red) - to $t=0$. (Color figure online)

In order to get the corresponding formulas for Dirichlet and Neumann boundary conditions it suffices to replace in (37) the distance $D$ by $2 D$.

At $T=0$ the correlation function equals

$$
\chi^{F}(r)=-\frac{1}{\left(2 \pi^{2}\right)^{2}}\left[\frac{\sin \left(k_{F} r\right)}{r^{3}}-\frac{k_{F} \cos \left(k_{F} r\right)}{r^{2}}\right]^{2}
$$

and decays $\sim r^{-4}$ with oscillations.

\section{Concluding Comments}

We have discussed the relation between the decay lengths characterizing the Casimir forces and the bulk correlation lengths in the case of ideal quantum gases enclosed in a slit with two infinite parallel walls separated by distance $D$. Both bosons and fermions were considered under different boundary conditions (periodic, Dirichlet, and Neumann) imposed at the walls.

Within a microscopic approach we have derived a basic formula relating the Casimir force to the one-particle density matrix. Its structure is the same for bosons and for fermions, Eqs. (22) and (23). In the case of ideal quantum gases the one-particle density matrix is directly related to the correlation function. It is well known that the choice of the boundary conditions influences the Casimir force while the bulk correlation function remains insensitive to this choice. The general structure of our basic formula does not depend on the boundary conditions which, however, dictate the choice of the arguments of the functions involved in it. That is how the influence of the boundary conditions on Casimir forces is reflected in Eqs. (22) and (23).

The case of the ideal Bose gas is particularly interesting due to the Bose-Einstein condensation which takes place at $\mu=0$. For thermodynamic states corresponding to the phase containing no condensate and in the limit $\mu \rightarrow 0$ the Casimir force decays exponentially with the decay length $\kappa^{B}$ depending on the chosen boundary conditions. We checked that $\kappa_{D i r}^{B}=\kappa_{N e u}^{B}=$ $\kappa_{\text {per }}^{B} / 2=\xi^{B} \propto(-\mu)^{-1 / 2}$, where $\xi^{B}$ denotes the correlation length of an ideal Bose gas and 
the proportionality to $(-\mu)^{-1 / 2}$ holds at fixed temperature $T<T_{c}$.

Analogous relations hold for ideal Fermi gas. Although in this case there is neither phase transition nor critical point, the Casimir force and the bulk correlation function display exponentially damped oscillations whose amplitude increases in the limit $T \rightarrow 0$. These oscillations correspond to the change of sign of the Casimir force: upon increasing the distance between the walls it becomes in turn attractive and repulsive. One can identify the corresponding decay length $\kappa^{F}$ and its relation to the fermionic bulk correlation length $\xi^{F}$. It turns out that these relations are the same as in the case of bosons, i.e., $\kappa_{\text {Dir }}^{F}=\kappa_{N e u}^{F}=$ $\kappa_{p e r}^{F} / 2=\xi^{F} \propto T^{-1}$, where the proportionality to $T^{-1}$ holds at fixed density $\rho$.

Open Access This article is distributed under the terms of the Creative Commons Attribution License which permits any use, distribution, and reproduction in any medium, provided the original author(s) and the source are credited.

\section{References}

1. Ziff, R.M., Uhlenbeck, G., Kac, M.: The ideal Bose-Einstein gas, revisited. Phys. Rep. 32, 169-248 (1977)

2. Subrahmanyam, V., Barma, M.: Finite-size effects in the ideal Fermi gas. J. Phys. A 22, L489-496 (1989)

3. Krech, M.: Casimir Effect in Critical Systems. World Scientific, Singapour (1994)

4. Lee, M.H., Long, M.: Off-diagonal long-range order, pair distribution function, and structure factor of the ideal Fermi gas in D dimensions and Prices inequality. Phys. Rev. E 52, 185-189 (1995)

5. Kardar, M., Golestanian, R.: The friction of vacuum, and other fluctuation-induced forces. Rev. Mod. Phys. 71, 1233-1245 (1999)

6. Zagrebnov, V.A., Bru, J.-B.: The Bogoliubov model of weakly imperfect Bose gas. Phys. Rep. 350, 291-434 (2001)

7. Bordag, M., Mohideen, U., Mostepanienko, V.M.: New developments in the Casimir effect. Phys. Rep. 353, 1-205 (2001)

8. Martin, P.A., Zagrebnov, V.A.: The Casimir effect for the Bose-gas in slabs. Europhys. Lett. 73, 15-20 (2006)

9. Gambassi, A., Dietrich, S.: Comment on "The Casimir effect for the Bose-gas in slabs" by P. A. Martin and V. A. Zagrebnov. Relation between the thermodynamic Casimir effect in Bose-gas slabs and critical Casimir forces. Europhys. Lett. 74, 754-755 (2006)

10. Kolomeisky, E.B., Straley, J.P., Timmins, M.: Casimir effect in a one-dimensional gas of free fermions. Phys. Rev. A 78, 022104 (2008)

11. Su, G.-Z., Ou, C.-J., Wang, Q.-P., Chen, J.-C.: Finite-size effects in a D-dimensional ideal Fermi gas. Chin. Phys. B 18, 5189-5195 (2009)

12. Bosse, J., Pathak, K.N., Singh, G.S.: Analytical pair correlations in ideal quantum gases: temperaturedependent bunching and antibunching. Phys. Rev. E 84, 042101 (2011)

13. Napiórkowski, M., Piasecki, J.: Casimir force induced by an imperfect Bose gas. Phys. Rev. E 84, 061105 (2011)

14. Chaba, A.N., Pathria, R.K.: Evaluation of lattice sums using Poisson's summation formula. II. J. Phys. A 9, 1411-1423 (1976)

15. Napiórkowski, M., Piasecki, J.: The bulk correlation length and the range of thermodynamic Casimir forces at Bose-Einstein condensation. J. Stat. Phys. 147, 1145-1155 (2012)

16. Chen, L.-W., Su, G.-Z., Chen, J.-C., Andresen, B.: Oscillating Casimir force between two slabs in a Fermi sea. Chin. Phys. B 21, 010501 (2012)

17. Jakubczyk, P., Nowak, K.: The imperfect Bose gas in D dimensions: critical behavior and Casimir forces. J. Stat. Mech. 2013, P06015 (2013)

18. Isihara, A.: Statistical Physics, vol. 11. Academic Press, Inc., London (1971)

19. Buffet, E., Pule, J.V.: Fluctuation properties of the imperfect Bose gas. J. Math. Phys. 24, 1608-1616 (1983)

20. van der Berg, M., Lewis, J.T., de Smedt, P.: Condensation in the imperfect Boson gas. J. Stat. Phys. 37, 697-707 (1984) 
21. Mostepanienko, V.M., Trunov, N.N.: The Casimir Effect and its Applications. Clarendon Press, Oxford (1997)

22. Levin, M., MaCauley, A.P., Rodriguez, A.W., Reid, M.T.H., Johnson, S.G.: Casimir repulsion between metallic objects in vacuum. Phys. Rev. Lett. 105, 090403 (2010)

23. Hertlein, C., Helden, L., Gambassi, A., Dietrich, S., Bechinger, C.: Direct measurement of critical Casimir forces. Nature 451, 172-175 (2008) 